\title{
Trabalho sexual em período de pandemia por COVID-19 no contexto íbero-americano: análise de anúncios em websites
}

\author{
Sex Work during the COVID-19 Pandemic Period in the Ibero- \\ American Context: analysis of advertisements on websites
}

Taciana Silveira Passos (https://orcid.org/0000-0002-5312-095X) ${ }^{1}$

Marcos Antonio Almeida-Santos (https://orcid.org/0000-0003-0622-6257) ${ }^{1}$
${ }^{1}$ Pós-Graduação em Saúde e Ambiente Universidade Tiradentes. Av. Murilo Dantas 300, Farolândia. 49032490 Aracaju SE Brasil. tacianasilveirapassos@ gmail.com

\begin{abstract}
Sex workers become increasingly economically vulnerable due to the restrictive measures implemented to combat the coronavirus pandemic. In this respect, the scope of this study is to analyze the content of prostitution websites and advertisements regarding measures related to the COVID-19 pandemic. It involved a description of the visits and analysis of content of communications on websites that advertise commercial sex transactions. The percentage change in the number of visits for three periods from 02/2019 to 04/2020 was calculated. Subsequent$l y$, ads with the terms "corona," "pandemic" and "quarantine" on websites that offer search engines were extracted. The Bardin method was then used for content analysis. There was an increase in the number of visits to prostitution websites between 2019 and 2020, followed by a decrease with the advent of the coronavirus pandemic crisis. With regard to the protection measures during the pandemic, health recommendations and the incentive to engage in virtual sex are highlighted. Of the 1,991,014 advertisements, $0.51 \%$ mention the COVID-19 crisis regarding noncompliance with social distancing, protection measures and the offer of online sex.

Key words Sex work, Advertisements, Social media, Coronavirus infections, Pandemics
\end{abstract}

Resumo Trabalhadores do sexo tornam-se cada vez mais vulneráveis economicamente como resultado das medidas restritivas implementadas para responder à pandemia de coronavírus. Nesse sentido, o objetivo deste estudo é analisar o conteúdo dos websites e anúncios de prostituição sobre medidas relacionadas à pandemia por COVID-19. Trata-se de descrição do fluxo de visitas e análise de conteúdo das comunicações em websites que anunciam transações de sexo comercial. Realizou-se cálculo de variação percentual do número de visitas para três períodos compreendidos entre 02/2019 a 04/2020. Posteriormente, extraíram-se anúncios com os termos "corona", "pandemia" $e$ "quarentena" em websites que oferecem mecanismo de busca. Para análise de conteúdo, utilizou-se o método de Bardin. Houve aumento no número de acessos nos websites de prostituição entre o ano de 2019 e 2020, seguido de queda com a advento da crise pandêmica por coronavírus. Dentre as medidas de proteção durante a pandemia, destacam-se as recomendações de saúde e o incentivo ao sexo virtual. Dentre 1.991.014 anúncios, 0,51\% mencionam a crise por COVID-19 quanto ao descumprimento do distanciamento social, medidas de proteção e oferta de sexo on-line.

Palavras-chave Trabalho sexual, Anúncios, Mídias sociais, Infecções por Coronavírus, Pandemias 


\section{Introdução}

A comunicação digital gerou impacto na forma como os indivíduos organizam suas vidas, conduzem os relacionamentos e realizam as transações comerciais. Em relação ao trabalho sexual, houve migração para plataformas on-line, com consequente declínio dos dois mercados tradicionalmente predominantes: os de rua e as instalações gerenciadas para comércio do sexo sob a forma de bordéis, apartamentos ou saunas ${ }^{1-4}$. A indústria do sexo operada através de tecnologias virtuais é representada por serviços de venda on-line mediante webcam, venda de fotos e vídeos; ou publicidade, marketing e organização do trabalho para facilitar os serviços e organizar encontros off-line - ou seja, presenciais ${ }^{5,6}$.

Durante a pandemia da síndrome respiratória aguda grave por coronavírus (SARS-CoV-2), essas atividades presenciais a dois, sexo grupal e a oferta de festas sexuais com uso de substâncias químicas podem resultar em aglomerados de transmissão ${ }^{7}$. Nesse sentido, os riscos relacionados ao trabalho sexual tendem a afetar mais pessoas do que as diretamente envolvidas nas interações.

Em 24 de março de 2020, a pandemia da SARS-CoV-2 afetou quase 400.000 pessoas em 168 países nos cinco continentes. Pacientes idosos (> 60 anos) e com comorbidades (por exemplo, hipertensão, diabetes, doença cardiovascular, doença pulmonar e doença renal crônica) apresentam infecção mais grave e pior prognóstico ${ }^{8}$.

Dado que inúmeros processos sociais influenciam a prostituição, o que ocorre em outros setores da sociedade impacta nas demais dimensões ${ }^{9-12}$. No âmbito da saúde pública, é necessário investigar o andamento dos serviços oferecidos por parte deste coletivo durante a crise pandêmica de COVID-19, uma vez que existe variabilidade dos serviços (online e offline) com diferentes formas organizacionais.

O COVID-19 tem apresentado efeitos significativos na renda das profissionais do sexo e, como tal, causa estresse adicional ${ }^{13-15}$. Além disso, a rotina de vida laboral e o medo do estigma podem distanciar essa população dos serviços de saúde ${ }^{16,17}$.

Em busca realizada nas bases de dados Pubmed, SciELO e Google Scholar, nos idiomas inglês, português e espanhol, até o dia 19 de maio de 2020, não foram encontrados registros de publicação sobre o funcionamento do trabalho sexual em tempos de COVID-19 na América Latina. Portanto, o objetivo desse estudo é analisar o conteúdo dos websites e dos anúncios de prostituição sobre medidas relacionadas à pandemia por COVID-19. Optou-se por realizar a análise em países da América Latina (países da América cuja língua falada deriva do latim) e Europa Meridional devido ao grande fluxo migratório com fins de prostituição e turismo sexual no contexto ibero-americano ${ }^{18,19}$.

\section{Metodologia}

$\mathrm{O}$ estudo segue delineamento exploratório e descritivo com abordagem quanti-qualitativa de fluxo de visitas e comunicações em websites que anunciam transações de sexo comercial. Trata-se de recorte de tese de doutorado em andamento, com projeto aprovado por Comitê de Ética em Pesquisa.

$\mathrm{O}$ advento das mídias sociais revelou, inclusive, várias oportunidades para a adoção da pesquisa virtual. As diretrizes para adaptar as técnicas de coleta e as análises de dados presenciais às novas contingências das comunicações culturais mediadas por computador foram identificadas por Kozinets ${ }^{20}$ nas seguintes fases relacionadas ao planejamento da pesquisa: reconhecimento do espaço virtual, coleta de dados, interpretação dos dados, garantia de padrões éticos e representação da pesquisa.

Nesse sentido, a pesquisa deu-se início através de mapeamento das plataformas existentes. Os países e websites de prostituição foram escolhidos de acordo com o critério de posição no Top Websites Ranking apresentado no site SimilarWeb (Tabela 1). Este site de Search Engine Optimization (SEO) calcula automaticamente o tráfego orgânico estimado de um website com base em classificações. Todas as visitas que um canal digital (como o site ou blog de uma empresa) recebe são chamadas de tráfego. $O$ tráfego orgânico são as visitas conquistadas de maneira espontânea, sem usar anúncios. Geralmente esse tipo de tráfego vem de mecanismos de busca, como o Google e o Bing.

O SimilarWeb também foi utilizado para obter informações sobre o número de visitas de cada website em três períodos diferentes: Primeiro período $(\mathrm{T} 01)=$ soma do total de visitas nos meses de fevereiro a abril de 2019 - coleta de dados realizada no dia 21/05/2019. Segundo período $(\mathrm{T} 02)=$ soma do total de visitas nos meses de novembro de 2019 a janeiro de 2020 - coleta de dados realizada no dia 17/05/2020. Terceiro período $(\mathrm{T} 03)=$ soma do total de visitas nos meses 
Tabela 1. Descrição dos três principais sites de anúncio de prostituição de países da América Latina e Europa Meridional.

\begin{tabular}{|c|c|c|c|c|}
\hline País de origem & Domínio & $\begin{array}{l}\text { Posição } \\
\text { por país }\end{array}$ & $\begin{array}{l}\text { Ranking } \\
\text { global }^{\star}\end{array}$ & $\begin{array}{c}\text { Ranking na } \\
\text { categoria Adulto* }^{\star}\end{array}$ \\
\hline \multirow[t]{3}{*}{ Argentina } & distintas.net & $1^{\circ}$ & $39.937^{\circ}$ & $4.063^{\circ}$ \\
\hline & foroescortsar.com & $2^{o}$ & $64.309^{\circ}$ & $6.212^{\circ}$ \\
\hline & bairesgirls.net & $3^{\circ}$ & $74.691^{\circ}$ & $7.075^{\circ}$ \\
\hline \multirow[t]{3}{*}{ Brasil } & fatalmodel.com & $1^{\circ}$ & $1.673^{\circ}$ & $107^{\circ}$ \\
\hline & photoacompanhantes.com & $2^{o}$ & $2.414^{\circ}$ & $160^{\circ}$ \\
\hline & garotacomlocal.com & $3^{\circ}$ & $15.853^{\circ}$ & $1.057^{\mathrm{o}}$ \\
\hline \multirow[t]{3}{*}{ Chile } & tusamantes.cl & $1^{\circ}$ & $64.407^{\circ}$ & $6.217^{\circ}$ \\
\hline & laestokada.cl & $2^{\circ}$ & $81.929^{\circ}$ & $7.646^{\circ}$ \\
\hline & relaxchile.cl & $3^{\circ}$ & $102.695^{\circ}$ & $9.188^{\circ}$ \\
\hline \multirow[t]{3}{*}{ Colômbia } & photoprepagos.com & $1^{\mathrm{o}}$ & $36.022^{\circ}$ & $3.688^{\circ}$ \\
\hline & pasionprepagos.com & $2^{\circ}$ & $120.544^{\circ}$ & $10.546^{\circ}$ \\
\hline & prepagos69.com & $3^{\circ}$ & $153.592^{\circ}$ & $12.923^{\circ}$ \\
\hline \multirow[t]{3}{*}{ Espanha } & pasion.com & $1^{\mathrm{o}}$ & $3.124^{\circ}$ & $217^{\circ}$ \\
\hline & nuevoloquo.com & $2^{o}$ & $18.693^{\circ}$ & $2.370^{\circ}$ \\
\hline & slumi.com & $3^{\circ}$ & $27.166^{\circ}$ & $2.919^{\circ}$ \\
\hline \multirow[t]{3}{*}{ França } & sexemodel.com & $1^{\mathrm{o}}$ & $3.152^{\circ}$ & $219^{\circ}$ \\
\hline & ladyxena.com & $2^{\circ}$ & $36.929^{\circ}$ & $2.407^{\circ}$ \\
\hline & 6annonce.com & $3^{\circ}$ & $39.769^{\circ}$ & $2.560^{\circ}$ \\
\hline \multirow[t]{3}{*}{ Itália } & moscarossa.biz & $1^{\mathrm{o}}$ & $22.535^{\circ}$ & $1.493^{\circ}$ \\
\hline & escortforumit.xxx & $2^{\circ}$ & $23.914^{\circ}$ & $1.586^{\circ}$ \\
\hline & escort-advisor.com & $3^{\circ}$ & $26.388^{\circ}$ & $1.749^{\circ}$ \\
\hline \multirow[t]{3}{*}{ México } & mileroticos.com & $1^{\circ}$ & $2.118^{\circ}$ & $232^{\circ}$ \\
\hline & sustitutas.com & $2^{\circ}$ & $62.210^{\circ}$ & $6.038^{\circ}$ \\
\hline & laboutique.vip & $3^{\circ}$ & $83.561^{\circ}$ & $7.762^{\circ}$ \\
\hline \multirow[t]{3}{*}{ Peru } & photokinesiologas.com & $1^{\circ}$ & $19.978^{\circ}$ & $1.462^{\circ}$ \\
\hline & perutops.com & $2^{\circ}$ & $54.981^{\circ}$ & $5.425^{\circ}$ \\
\hline & peru-amateur.com & $3^{\circ}$ & $97.825^{\circ}$ & $8.792^{\circ}$ \\
\hline \multirow[t]{3}{*}{ Portugal } & classificadosx.net & $1^{\mathrm{o}}$ & $23.123^{\circ}$ & $1.115^{\circ}$ \\
\hline & rua69.com & $2^{\circ}$ & $80.297 \circ$ & $5.050^{\circ}$ \\
\hline & apartadox.com & $3^{\circ}$ & $164.597^{\circ}$ & $9.394^{\circ}$ \\
\hline
\end{tabular}

${ }^{\star}$ Dados obtidos no SimilarWeb LTD (site de de Search Engine Optimization) no dia 21/05/2019.

Nota: Os países foram selecionados de acordo com a posição dos respectivos sites no Ranking Global.

de fevereiro a abril de 2019 - coleta de dados realizada no dia 17/05/2020.

Através desses dados, foi realizado o cálculo de variação percentual entre os períodos (Equação 01), para analisar se houve mudanças no tráfego no período da pandemia.

$$
\frac{\text { Valor atual - Valor antigo }}{\text { Valor antigo }} \times 100
$$

Para a análise de conteúdo dos sites foram extraídas as comunicações em relação à pandemia por COVID-19 (tais como informações, recomendações e restrições) através de links, homepages e posts publicados. Devido à potencial natureza temporária dos anúncios da Internet, também foram capturadas imagens através da ferramenta Print Screen.

Em seguida, dividimos as informações em três categorias: informações sobre auxílios financeiros; recomendações sobre prevenção do contágio; estratégias como destaques para a oferta de sexo virtual (apenas sexo na modalidade online via webcam, áudios e/ou mensagens de texto) ou novas plataformas de oferta de sexo via webcam.

$\mathrm{Na}$ coleta de dados da segunda etapa do estudo, foi analisada a proporção de anúncios de trabalhadores sexuais que mencionaram a pandemia por Covid 19. Esses dados só foram possíveis de 
serem coletados em websites que fornecem recurso de busca para termos inseridos nas comunicações das propagandas (Tabela 1). Assim, os dados foram obtidos tendo-se considerado as análises por: termos de pesquisa ("corona"); ("quarentena"); ("pandemia"). Os termos foram traduzidos no idioma de cada país e utilizados nos respectivos motores de busca (search engines).

Posteriormente, extraíram-se os anúncios publicados na primeira página dos websites. Devido à natureza rotativa e mutável dos anúncios, utilizamos o recurso de impressão Microsoft Print to PDF para cada termo de pesquisa em cada website. Com esses textos selecionados, utilizamos o método da análise de conteúdo de Bardin $^{21}$ para estudar três categorias: total descumprimento sanitário, minimização dos riscos e atendimento sem contato presencial. Os discursos foram traduzidos para o idioma português como medida de padronização para comparações entre países de diferentes línguas.

Apenas foram utilizados os sites de acesso aberto para qualquer indivíduo maior de 18 anos. O consentimento não foi necessário, pois a pesquisa acontece com descarregamento de mensagens em websites comerciais, não ocorrendo intervenção ou interação. $\mathrm{O}$ anonimato dos indivíduos que escreveram os anúncios foi mantido. Os documentos em PDF e as impressões dos websites estão armazenados em pasta protegida com palavra-chave.

\section{Resultados}

De acordo com as buscas realizadas nos dias 21/05/2019 e 17/05/2020 (Tabela 2), o trabalho sexual é cada vez mais comercializado em websites. Destacou-se o crescimento expressivo no Brasil e Colômbia. No entanto, houve queda no número de visitas na maioria dos sites entre fevereiro a abril de 2020, trimestre de andamento da pandemia COVID-19.

A principal estratégia dos websites para continuar o mercado sexual minimizando riscos para trabalhadores e clientes foi a oferta de novos links, ou link antigo posto em destaque, para acesso aos serviços de sexo on-line através de webcam, que também é chamado de Cybervoyerismo. Comunicações desse tipo foram encontradas em sites da Argentina, Chile, Espanha, França, Itália e Portugal. Nenhum dos principais sites brasileiros, colombianos, mexicanos e peruanos apresentaram esse tipo de marketing, tampouco apresentaram alguma estratégia (Quadro 1).
Ao menos um dos três principais sites de cada país europeu apresentou posts e/ou avisos com informações sanitárias e recomendações de prevenção. Na América Latina, foram encontradas informações sanitárias em sites da Argentina, Chile, Colômbia e México (Figura 1; Quadro 1).

Dentre as medidas recomendadas pelos websites para profissionais que ainda estavam realizando atendimento presencial, as mais citadas foram: evitar contato íntimo com pessoas que apresentem sintomas de gripe (especialmente tosse ou febre); medir temperatura do cliente; exigir higiene; lavar todo o corpo após relação sexual, especialmente as mãos com sabão de banho; evitar o contato entre as faces; recomendação da posição do Kama Sutra denominada "cachorrinho" (indivíduo receptivo de bruços, em posição mais inclinada, quase com o rosto no colchão) pois possui a maior distância entre os rostos; não trocar saliva e ter cuidado com os olhos; uso do preservativo em sexo oral; oferta de chat para compartilhar informações sobre clientes que não respeitam as regras, sintomáticos ou contaminados.

Nenhum site brasileiro e peruano apresentou informações sobre auxílios sociais ou informações sanitárias. O quesito de informações sobre auxílios sociais foi o mais negligenciado pelos sites, apenas um site chileno e um português ofereceram alguma abordagem nesse sentido (Quadro 1).

O site chileno (laestokada.cl) oferece gratuidade no serviço de publicação do anúncio como uma forma de auxílio nesse período de pandemia e até mesmo ampliação de acesso a outros trabalhadores sexuais de rua e/ou bordéis, por exemplo. Enquanto o site português (classificadosx. net) oferece um blog com matéria intitulada Precisas de ajuda? Vê que tipo de apoios podes receber. $\mathrm{Na}$ referida matéria pode-se encontrar números e e-mails de Emergência Social, Serviço Social Público e Associações/Grupos da sociedade civil.

Referente ao conteúdo dos anúncios, a Tabela 3 ilustra a distribuição dos termos "corona", "quarentena" e "pandemia" nos websites, sendo que foram excluídos alguns que possuem ferramenta de busca apenas para nomes de cidades, nomes de profissionais e números de telefone, e outros que não possuem nenhum mecanismo. Dentre quase dois milhões de anúncios publicados nos referidos sites, apenas $0,51 \%$ apresentaram comunicações sobre o COVID-19. O termo "quarentena" é o mais utilizado (77,58\%).

Considerando os elementos textuais e visuais dos anúncios, não se pode realizar uma análise consistente relacionada aos aspectos socioeconô- 
Tabela 2. Variação percentual do número de visitas nos websites de prostituição em três períodos, entre fevereiro de 2019 a abril de 2020 .

\begin{tabular}{|c|c|c|c|c|c|c|}
\hline \multirow[b]{2}{*}{$\begin{array}{l}\text { País de } \\
\text { origem }\end{array}$} & \multirow[b]{2}{*}{ Domínio } & \multicolumn{5}{|c|}{ Total de visitas } \\
\hline & & $\begin{array}{c}\text { T01 }^{*} \\
02 / 2019 \\
- \\
04 / 2019\end{array}$ & $\begin{array}{c}\text { T02† } \\
11 / 2019 \\
- \\
01 / 2020\end{array}$ & $\begin{array}{c}\text { VP } \\
\text { (T01-T02) }\end{array}$ & $\begin{array}{c}\text { T03† } \\
02 / 2020 \\
- \\
04 / 2020\end{array}$ & $\begin{array}{c}\text { VP } \\
\text { (T02-T03) }\end{array}$ \\
\hline \multirow[t]{3}{*}{ Argentina } & distintas.net & 2.377 .000 & 2.570 .000 & $08 \%$ & 2.740 .000 & $07 \%$ \\
\hline & foroescortsar.com & 1.844 .400 & 1.920 .000 & $04 \%$ & 1.620 .000 & $-16 \%$ \\
\hline & bairesgirls.net & 1.024 .500 & 920.000 & ${ }^{-} 10 \%$ & 650.000 & $-29 \%$ \\
\hline \multirow[t]{3}{*}{ Brasil } & fatalmodel.com & 39.596 .900 & 59.100 .000 & $49 \%$ & 50.400 .000 & $-15 \%$ \\
\hline & photoacompanhantes.com & 23.980 .600 & 37.800 .000 & $58 \%$ & 33.700 .000 & ${ }^{-} 11 \%$ \\
\hline & garotacomlocal.com & 6.540 .400 & 11.950 .000 & $83 \%$ & 8.650 .000 & $-28 \%$ \\
\hline \multirow[t]{3}{*}{ Chile } & tusamantes.cl & 1.491 .000 & 1.270 .000 & ${ }^{-} 15 \%$ & 910.000 & $-28 \%$ \\
\hline & laestokada.cl & 1.358 .100 & 1.320 .000 & $-03 \%$ & 1.280 .000 & $-03 \%$ \\
\hline & relaxchile.cl & 1.251 .400 & 451.742 & $-64 \%$ & 370.589 & $-18 \%$ \\
\hline \multirow[t]{3}{*}{ Colômbia } & photoprepagos.com & 2.491 .200 & 4.500 .000 & $81 \%$ & 3.390 .000 & $-25 \%$ \\
\hline & pasionprepagos.com & 754.000 & 1.460 .000 & $94 \%$ & 1.220 .000 & $-16 \%$ \\
\hline & prepagos69.com & 503.300 & 950.000 & $89 \%$ & 725.000 & ${ }^{-} 15 \%$ \\
\hline \multirow[t]{3}{*}{ Espanha } & pasion.com & 62.500 .400 & 61.800 .000 & $-01 \%$ & 46.300 .000 & $-25 \%$ \\
\hline & nuevoloquo.com & 3.008 .700 & 5.000 .000 & $66 \%$ & 3.490 .000 & $-30 \%$ \\
\hline & slumi.com & 2.970 .200 & 5.450 .000 & $83 \%$ & 3.290 .000 & $-40 \%$ \\
\hline \multirow[t]{3}{*}{ França } & sexemodel.com & 36.642 .200 & 39.200 .000 & $07 \%$ & 31.400 .000 & $-20 \%$ \\
\hline & ladyxena.com & 6.062 .400 & 6.250 .000 & $03 \%$ & 5.050 .000 & $-19 \%$ \\
\hline & 6annonce.com & 5.985 .000 & 6.750 .000 & $13 \%$ & 4.850 .000 & $-28 \%$ \\
\hline \multirow[t]{3}{*}{ Itália } & moscarossa.biz & 13.642 .200 & 14.550 .000 & $07 \%$ & 9.200 .000 & $-37 \%$ \\
\hline & escortforumit.xxx & 9.362 .400 & 9.600 .000 & $03 \%$ & 6.350 .000 & $-34 \%$ \\
\hline & escort-advisor.com & 9.180 .000 & 1.1095 .000 & $21 \%$ & 7.900 .000 & $-29 \%$ \\
\hline \multirow[t]{3}{*}{ México } & mx.mileroticos.com & 39.086 .600 & 54.400 .000 & $39 \%$ & 47.400 .000 & $-13 \%$ \\
\hline & mx.sustitutas.com & 1.770 .900 & 2.110 .000 & $19 \%$ & 1.710 .000 & $-19 \%$ \\
\hline & laboutique.vip & 1.159 .300 & 1.000 .000 & ${ }^{-} 14 \%$ & 990.000 & $-01 \%$ \\
\hline \multirow[t]{3}{*}{ Peru } & photokinesiologas.com & 4.851 .800 & 7.000 .000 & $44 \%$ & 5.450 .000 & $-22 \%$ \\
\hline & perutops.com & 2.013 .400 & 2.220 .000 & $10 \%$ & 2.130 .000 & $-04 \%$ \\
\hline & peru-amateur.com & 912.300 & 750.000 & $-18 \%$ & 540.000 & $-28 \%$ \\
\hline \multirow[t]{3}{*}{ Portugal } & classificadosx.net & 4.243 .400 & 7.000 .000 & $65 \%$ & 5.900 .000 & ${ }^{-} 16 \%$ \\
\hline & rua69.com & 1.804 .100 & 2.290 .000 & $27 \%$ & 1.650 .000 & $-28 \%$ \\
\hline & apartadox.com & 1.473 .000 & 1.090 .000 & $-26 \%$ & 860.000 & $-21 \%$ \\
\hline
\end{tabular}

$\mathrm{VP}=$ Variação Percentual. ${ }^{*}$ Dados obtidos no @ SimilarWeb LTD (site de de Search Engine Optimization) no dia 21/05/2019. $\dagger$ Dados obtidos no @ SimilarWeb LTD (site de de Search Engine Optimization) no dia 17/05/2020.

micos e diversidade de gênero. Ainda assim, podese mencionar que não foram observadas diferenças entre raça ou nacionalidade, mas os anúncios eram em sua maioria de mulheres cisgênero. $\mathrm{O}$ espírito empreendedor, além de recursos e conhecimentos necessários para realizar publicidade na Internet, pode sugerir a necessidade de um certo grau de capital cultural e privilégio educacional.

Ao analisar o conteúdo da primeira página de anúncios dos websites, observou-se que as comunicações sobre COVID-19 nem sempre ressaltavam medidas de proteção. Algumas vezes, isso representava a oferta de descumprimento de medidas recomendadas pela Organização Mundial de Saúde. A oferta de sexo presencial varia entre 15 a 150 dólares por hora, aproximadamente. Comumente, expressões relacionadas ao rompimento da quarentena eram utilizadas com o intuito de proporcionar alívio de tensão e entretenimento mediante serviços sexuais:

"Sou um homem, procuro mulheres que queiram passar um momento gostoso nesta quarentena e queiram desestressar" (tusamantes.cl, Viña del Mar - Chile, 35 anos). 
Quadro 1. Análise de conteúdo dos websites de prostituição quanto ao tema Covid-19.

\begin{tabular}{|c|c|c|c|c|}
\hline País & Domínio & Auxílios Sociais & Recomendações sanitárias & Estratégias \\
\hline \multirow[t]{3}{*}{ Argentina } & distintas.net & - & - & $\begin{array}{l}\text { Link novo para sexo via } \\
\text { plataforma online }\end{array}$ \\
\hline & foroescortsar.com & - & $\begin{array}{l}\text { Post recomenda seguir as } \\
\text { indicações governamentais }\end{array}$ & $\begin{array}{l}\text { Link novo para sexo via } \\
\text { plataforma online }\end{array}$ \\
\hline & www.bairesgirls.net & - & $\begin{array}{l}\text { Incentivo aos clientes para } \\
\text { realização de sexo virtual }\end{array}$ & $\begin{array}{l}\text { Link novo para sexo via } \\
\text { plataforma online }\end{array}$ \\
\hline \multirow[t]{3}{*}{ Brasil } & fatalmodel.com & - & - & - \\
\hline & $\begin{array}{l}\text { photoacompanhantes. } \\
\text { com }\end{array}$ & - & - & - \\
\hline & garotacomlocal.com & - & - & - \\
\hline \multirow[t]{3}{*}{ Chile } & tusamantes.cl & - & - & - \\
\hline & laestokada.cl & $\begin{array}{l}\text { Publicação grátis } \\
\text { devido à crise }\end{array}$ & - & $\begin{array}{l}\text { Link novo para sexo via } \\
\text { plataforma online }\end{array}$ \\
\hline & relaxchile.cl & - & - & $\begin{array}{l}\text { Link destaque para sexo } \\
\text { via plataforma online }\end{array}$ \\
\hline \multirow[t]{3}{*}{ Colômbia } & photoprepagos.com & - & - & - \\
\hline & pasionprepagos.com & - & $\begin{array}{l}\text { Recomendações de proteção } \\
\text { em atendimentos presenciais }\end{array}$ & - \\
\hline & prepagos69.com & - & - & - \\
\hline \multirow[t]{3}{*}{ Espanha } & pasion.com & - & $\begin{array}{l}\text { Recomendações de } \\
\text { utilização da vídeo-chamada }\end{array}$ & - \\
\hline & nuevoloquo.com & - & $\begin{array}{l}\text { Link com matérias sobre } \\
\text { cuidados durante a pandemia }\end{array}$ & $\begin{array}{l}\text { Link destaque para sexo } \\
\text { via plataforma online }\end{array}$ \\
\hline & slumi.com & - & - & - \\
\hline \multirow[t]{3}{*}{ França } & sexemodel.com & - & $\begin{array}{l}\text { Incentivo aos clientes para } \\
\text { realização de sexo virtual }\end{array}$ & $\begin{array}{l}\text { Link novo para sexo via } \\
\text { plataforma online }\end{array}$ \\
\hline & ladyxena.com & - & - & - \\
\hline & 6annonce.com & - & - & $\begin{array}{l}\text { Link destaque para sexo } \\
\text { via plataforma online }\end{array}$ \\
\hline \multirow[t]{3}{*}{ Itália } & moscarossa.biz & - & - & \\
\hline & escortforumit.xxx & - & $\begin{array}{l}\text { Informações sobre medidas } \\
\text { de prevenção e notícias }\end{array}$ & $\begin{array}{l}\text { Link destaque para sexo } \\
\text { via plataforma online }\end{array}$ \\
\hline & escort-advisor.com & - & - & - \\
\hline \multirow[t]{3}{*}{ México } & mx.mileroticos.com & - & $\begin{array}{l}\text { Post recomenda seguir as } \\
\text { indicações governamentais }\end{array}$ & - \\
\hline & mx.sustitutas.com & - & - & - \\
\hline & laboutique.vip & - & - & - \\
\hline \multirow[t]{3}{*}{ Peru } & photokinesiologas.com & - & - & - \\
\hline & perutops.com & - & - & - \\
\hline & peru-amateur.com & - & - & - \\
\hline \multirow[t]{3}{*}{ Portugal } & classificadosx.net & $\begin{array}{l}\text { Oferece guia de } \\
\text { auxílios sociais }\end{array}$ & $\begin{array}{l}\text { Oferece blog com matérias } \\
\text { sobre medidas de prevenção }\end{array}$ & - \\
\hline & rua69.com & - & - & $\begin{array}{l}\text { Link destaque para sexo } \\
\text { via plataforma online }\end{array}$ \\
\hline & apartadox.com & - & - & - \\
\hline
\end{tabular}

"Para aqueles casais que querem experimentar coisas novas ou ter fantasias e esquecer a quarentena. Máxima discrição e educação. (...) Também estou procurando mulheres maduras para aliviar a quarentena" (pasion.com, Valencia - Espanha, 28 anos).”

"Em meu atendimento farei com você tudo o que a sua mulher não faz, tudo com bastante 
higiene e segurança. Venha quebrar a quarentena" (photoacompanhantes.com; Osasco - Brasil, 23 anos).

"Nesta quarentena, não fique entediado em sua casa, melhor venha nos visitar, faremos com que você tenha um tempo muito agradável e gostoso" (mileróticos.com; Cidade do México - México, 19 anos).

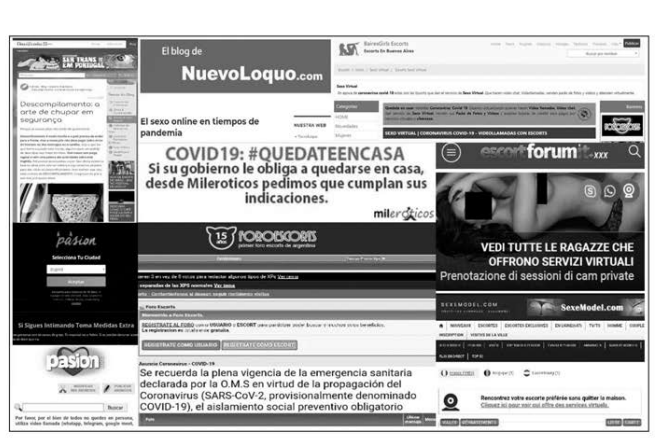

Figura 1. Mural com Prints dos websites Europeus e Américo-latinos que apresentaram informações, recomendações

e/ou restrições sanitárias.
"Entediado com a quarentena? Venha comigo e vamos fazer uma grande festa, com muito sexo sem limites, sexo grego e tudo o que sua cabecinha suja imagina farei com que se torne realidade. Eu vou até você, ou você pode vir ao meu apartamento" (newloquo.com; Barcelona - Espanha, 21 anos).

"Estou tomando todos os cuidados sobre o coronavírus, casa toda higienizada. Então venha tranquilo pois minha casa é um lugar seguro para você aproveitar e relaxar nesse momento tão difícil' (photoacompanhantes.com; São José dos Campos - Brasil, 26 anos).

Também foram encontradas estratégias de marketing como forma de potencializar o anúncio materializado em promoções para encontros presenciais, bem como oferta de sexo sem preservativo (al natural) e outras situações arriscadas, tais como sexo com cocaína (fiesta blanca). Além disso, os discursos costumam enfatizar a higiene do próprio corpo e do local de trabalho:

"Estou disponível apenas para clientes financeiramente solventes que desejam se divertir. (...) Por causa do coronavírus, não atendo a todos apenas os melhores" (newloquo.com; Lleida - Espanha, 25 anos)

"Realizo os meus trabalhos com local limpo e tranquilo, próximo da (...), venha aproveitar a

Tabela 3. Análise de conteúdo dos anúncios de prostituição quanto ao tema COVID-19.

\begin{tabular}{|c|c|c|c|c|c|c|}
\hline $\begin{array}{l}\text { País de } \\
\text { origem }\end{array}$ & Domínio & $\begin{array}{c}\text { "corona" } \\
(\mathrm{pt}) \\
\text { “coronavirus” } \\
(\mathrm{es} / \mathrm{fr} / \mathrm{it})\end{array}$ & $\begin{array}{l}\text { “quarentena" } \\
\text { (pt/it) } \\
\text { "cuarentena" } \\
(\text { es }) \\
\text { “quarantaine" } \\
\text { (fr) }\end{array}$ & $\begin{array}{l}\text { "pandemia" } \\
\text { (pt/es/it) } \\
\text { "pandémie" } \\
\text { (fr) }\end{array}$ & $\begin{array}{l}\text { Total de } \\
\text { anúncios }\end{array}$ & $\begin{array}{c}\text { Comunicações } \\
\text { COVID-19 }\end{array}$ \\
\hline Brasil & $\begin{array}{l}\text { photoacompanhantes. } \\
\text { com }\end{array}$ & 24 & 156 & 49 & 107.809 & $229(0,21 \%)$ \\
\hline Chile & tusamantes.cl & 11 & 24 & 18 & 59.264 & $53(0,09 \%)$ \\
\hline \multirow[t]{2}{*}{ Colômbia } & photoprepagos.com & 01 & 60 & 03 & 13.962 & $64(0,46 \%)$ \\
\hline & prepagos69.com & 0 & 12 & 0 & 340 & $12(3,53 \%)$ \\
\hline \multirow[t]{3}{*}{ Espanha } & pasion.com & 1.356 & 6.777 & 506 & 582.519 & $\begin{array}{r}8.639 \\
(1,48 \%)\end{array}$ \\
\hline & nuevoloquo.com & 51 & 71 & 0 & 175.000 & $122(0,07 \%)$ \\
\hline & slumi.com & 07 & 41 & 07 & 44.393 & $55(0,12 \%)$ \\
\hline França & sexemodel.com & 01 & 01 & 03 & 8020 & $05(0,06 \%)$ \\
\hline México & mx.mileroticos.com & 83 & 584 & 65 & 956.198 & $732(0,08 \%)$ \\
\hline Peru & photokinesiologas.com & 29 & 71 & 4 & 24.945 & $104(0,42 \%)$ \\
\hline \multirow[t]{2}{*}{ Portugal } & classificadosx.net & 30 & 73 & 28 & 16.760 & $131(0,78 \%)$ \\
\hline & rua69.com & 04 & 26 & 02 & 1804 & $32(1,77 \%)$ \\
\hline Total & & $\begin{array}{r}1.597 \\
(15,69 \%)\end{array}$ & $\begin{array}{r}7.896 \\
(77,58 \%) \\
\end{array}$ & $\begin{array}{r}685 \\
(6,73 \%) \\
\end{array}$ & 1.991 .014 & $\begin{array}{r}10.178 \\
(0,51 \%)\end{array}$ \\
\hline
\end{tabular}

pt = português; es = espanhol; fr = francês; it = italiano. 
minha promoção de quarentena, não perca essa oportunidade, beijos e até mais" (photoacompanhantes.com; São Paulo - Brasil, 23 anos).

"Aproveite minha promoção de quarentena, realizo adicional grátis: oral natural, atenção a casais, anal, sexo em trio" (photoprepagos.com, Bogotá - Colômbia, 23 anos).

"Venha eu espero por você. Farei tudo para você, exceto te passar coronavírus. Eu tenho tudo em dia tudo para que você fique tranquilo. Se você quiser eu até faço sexo natural" (tusamantes. cl, Santiago - Chile, 28 anos).

"Eu atendo, sem coronavírus, com todas as medidas de higiene e desinfecção. (...) Eu tenho um apartamento bonito com todos os tipos de comodidades e me desloco para hotel e casa. Eu amo beber e fiesta blanca" (pasion.com, Madri Espanha, 48 anos).

Outros profissionais do mercado sexual decidiram realizar o atendimento com algumas medidas de proteção:

"No meu atendimento rola massagem relaxante, oral sem finalização, vaginal, anal a combinar. Beijo na boca em época de coronavírus não rola. Sou muito higiênica" (photoacompanhantes.com; Belo Horizonte - Brasil, 24 anos).

"Ao chegar em minha casa, existem certos requisitos de higiene que devemos cumprir, tudo isso para nossos cuidados (devido ao coronavírus). Não é apenas passar gel antibacteriano. Não! Cuide-se bem! Minhas medidas de higiene são extremas, sempre dando o melhor dos melhores!" (mileróticos.com; Puebla - México, 22 anos).

"Agora, só faço sessões com máscaras até que o estado do alarme de coronavírus termine" (tusamantes.cl, Viña del Mar - Chile, 35 anos).

"Face ao estado de alerta devido ao novo coronavírus. Como trabalhadora do sexo para minimizarem riscos, só atendo nestas condições: 1 Tenho lençóis descartáveis, um por cada cliente. 2 - Toalhas lavadas para cada cliente. 3 - Obrigatório Tomar banho com água e sabão. 4 - Tenho álcool em gel para desinfetar antes e depois. 5 - Mantenho o ambiente bem ventilado. Valor Reduzido $60 €$. Atendo apenas duas pessoas por dia. Faz a tua marcação" (classificadosx.net; Massamá - Portugal, 29 anos).

"Para cuidar-nos durante esta pandemia, você deve ter com você as ferramentas de biossegurança, os banhos devem ser feitos antes para evitar o contágio" (photoprepagos.com, Bogotá - Colômbia, 43 anos).

A preços mais baixos, entre 10 a 15 dólares por hora, aproximadamente, muitos oferecem o serviço online através de webcam como forma de proteção contra o coronavírus:
"Para conscientizar as pessoas a ficarem em casa devido ao alto risco de contaminação pelo coronavírus, estou com atendimento virtual, uma maneira gostosa de curtir essa quarentena e ficar em casa sem perder toda a diversão, farei atendimento por chamadas de vídeos, do jeito que você quiser, você escolhe desde o que vou vestir, até o que vou fazer, pagamentos por transferência, depósito, boletos ou QR code" (photoacompanhantes.com; Teresina - Brasil, 25 anos).

"Olá, sou atleta, por motivos de coronavírus, ofereço telefonemas eróticos e vídeo chamadas, minhas fotos do anúncio são reais" (pasion.com, Madrid - Espanha, 44 anos).

"As redes não espirram meu amor, comigo não correrá o risco de contrair coronavírus, eu sou Hellen, uma sexy pré-paga com curvas ricas, pele de canela e cabelo preto bonito. Eu me caracterizo por ser uma garota muito amorosa, divertida e acolhedora. Ofereço a você uma sessão sensual e sexy de brincadeira on-line" (photoprepagos.com, Manizales - Colômbia, 25 anos).

"Neste momento, devido à emergência em que todos vivemos com o coronavirus, estou oferecendo apenas o serviço de vídeo-chamada onde podemos nos masturbar, sabendo que o sexo virtual não infecta. Quem está na rua cuida do seu. Se você gostar, depois da quarentena, ofereço meu serviço presencial" (photokinesiologas.com; Lima - Peru, 19 anos).

Com valores aproximados ainda mais baixos, entre 2 a 5 dólares, alguns oferecem o serviço de packs. São pacotes com registros multimídia, como fotos e vídeos, com conteúdo erótico (corpo nu, masturbação, sexo explícito etc.):

"Devido ao problema atual que estamos enfrentando, ofereço meus serviços virtuais. Meu pacote delicioso é composto por trinta fotos intimas e dois vídeos de seis minutos cada. Neles você pode ver meu rosto, meus seios, minha vagina e meu ânus. Nos meus vídeos você pode me ver masturbando. Além disso, para todas as pessoas que comprarem meu pack, darei mais trinta minutos de meu serviço pessoal quando o período de quarentena passar. A única coisa que peço é que não compartilhem minhas fotos ou vídeos" (photoprepagos.com, Neiva - Colômbia, 19 anos).

"Rei, por apenas 100 pesos mexicanos, leve minhas fotos mais quentes e alguns vídeos muito quentes. Aprecie meu pack me olhando completa e sem censura. Curvas que matam rei e uma bunda enorme. Pagamentos Oxxo. Meu serviço de acompanhante volta quando passar a pandemia (mileróticos.com; Xalapa - México, 19 anos).

"No momento, não vou a lugar nenhum, estou apenas atendendo por vídeo-chamadas de- 
vido à pandemia, onde podemos nos masturbar um com o outro. Além disso, ofereço um pack muito quente de fotos e vídeo. (photokinesiologas. com; Lima - Peru; 25 anos)

\section{Discussão}

Antes de uma vacina contra SARS-CoV-2 se tornar amplamente disponível, são necessárias várias medidas para controlar a pandemia de COVID-19. Por recomendação da Organização Mundial de Saúde, o distanciamento social é uma das melhores medidas para que as pessoas não entrem em contato com o coronavírus, reduzindo a velocidade do contágio ${ }^{22}$. Contudo, trabalhadores sexuais exercem a profissão em regime informal, e ficar em casa significa perder ou diminuir a renda necessária para pagar as contas e sobreviver. No caso específico do mercado sexual, em diversos países, a falta de trabalho se soma ainda ao estigma e abandono por parte do poder público em garantir direitos básicos para a categoria ${ }^{23,24}$.

Os principais discursos políticos governamentais de gerenciamento de riscos, impostos na pandemia do COVID-19, afirmam que é necessário tomar precauções, preparar-se para entrar e permanecer em confinamento até o surgimento de melhora nos indicadores de contágio populacional. No entanto, essas mensagens institucionais e as práticas associadas alcançam diretamente algumas categorias mais privilegiadas, isto é, aquelas com maior acesso a capital social, humano, financeiro e político ${ }^{25}$. Com efeito, as estratégias preventivas, necessariamente impostas pelo poder público para combater o avanço da pandemia, quando atingem diretamente uma categoria menos privilegiada, tal como a que atua em trabalhos sexuais, deflagram com maior intensidade preocupações quanto à sobrevivência durante situações de distanciamento social, inviabilização da oferta de serviços em virtude de recessão econômica, adoecimento, necessidade de submeter-se a isolamento compulsório ou fase de recuperação prolongada.

Trabalhadores do sexo estão em maior risco de problemas de saúde ${ }^{26}$, uso indevido de substâncias e violência ${ }^{27}$. A menos que os serviços sexuais offline retomem a operação normal em breve e as dificuldades financeiras sejam resolvidas, seus problemas de saúde tendem a se multiplicar. As agências de prostituição têm criticado a falta de ação para proteger as necessidades de saúde de profissionais do sexo durante a vigência da pandemia ${ }^{28}$.
Assim como outros trabalhadores autônomos, alguns profissionais do sexo estão expostos a riscos e sem suporte do tecido social que lhes permita aceitar com facilidade a perspectiva de recolher-se em quarentena para se proteger da COVID-19, além de proteger a comunidade em geral. Uma minoria aderiu à estratégia de sexo online, provavelmente pelo fato de necessitar trabalhar mais horas para arrecadar o mesmo valor do sexo offline ou por falta de recursos (qualidade de navegação de internet, câmera e acessórios). Diante desse cenário, estariam potencialmente mais vulneráveis as trabalhadoras sexuais idosas e aquelas que exercem a prostituição em zonas de rua.

A especificidade das comunidades marginalizadas pode ser negligenciada com declarações abrangentes sobre as dificuldades que as pessoas enfrentam ou uma abordagem única para o planejamento e a resposta à pandemia ${ }^{29}$. Em uma pesquisa que investigou o trabalho sexual após o terremoto de Canterbury ${ }^{30}$, descobriram que as trabalhadoras do sexo experimentavam deslocamento geográfico, social e de renda. No entanto, suas necessidades foram ignoradas quando a cidade foi reconstruída.

No presente estudo, observou-se que alguns websites compartilharam recomendações de segurança específicas aos profissionais do sexo que continuam exercendo a prostituição em modalidade presencial. Além disso, a estratégia adotada para incentivar o distanciamento social foi colocar em destaque ou criar um acesso novo para anúncios que oferecem sexo virtual. Os websites destinados à propaganda do mercado sexual funcionam muitas vezes como formas organizativas de governança de trabalhadores sexuais. Adicionalmente, pode ser a principal referência de informações sobre saúde, segurança e recursos sociais $^{3,10,11,31,32}$.

Ainda que seja a estratégia mais segura de continuar exercendo a profissão, observou-se que a troca do sexo offline por online traz um prejuízo financeiro. De toda forma, os dados da remuneração devem ser considerados com cautela. Em primeiro lugar, é provável que a renda proveniente de sexo por webcam varie bastante de um mês para o outro. Em segundo, os números são estimativas baseadas em informações heterogêneas e, às vezes, imprecisas ${ }^{33}$.

Destaca-se o descaso dos principais websites peruanos e brasileiros ao não oferecer nenhuma informação ou estratégia de trabalho para profissionais do sexo. Vale ressaltar que, pouco mais de dois meses após medidas restritivas na América 
Latina, depois do Brasil, o Peru é o segundo país latino-americano com mais casos de COVID-19 em termos absolutos, de acordo com a Universidade Johns Hopkins, que monitora o progresso da pandemia no planeta $^{34}$.

\section{Limitações e perspectivas}

Acerca de limitações do trabalho em questão, nossa amostra deriva de fonte secundária com informações limitadas ao que foi publicado em websites e anúncios de trabalhadores sexuais. Pesquisas adicionais são necessárias para obter uma perspectiva intersetorial completa sobre trabalho sexual baseado na Internet, especialmente em relação à raça, etnia e diversidade de gênero.

Não obstante, os resultados desta investigação poderão suscitar políticas com foco em campanhas de saúde pública voltadas aos indivíduos que estão envolvidos direta ou indiretamente com o trabalho sexual comercializado na internet. Após a pandemia, acredita-se que, à medida que a Internet continua a crescer, um número maior de profissionais do sexo provavelmente anunciará serviços via Internet. Portanto, deve-se considerar que as comunidades on-line relacionadas ao trabalho sexual são importantes veículos para se disponibilizar instruções e mensagens sobre saúde e segurança, além de representaram um espaço onde se pode alcançar subpopulações frequentemente excluídas ${ }^{35}$.

\section{Conclusão}

Os websites latino-americanos e ibéricos com finalidade de mercado sexual tiveram uma experiência de ascensão no número de acesso entre o ano de 2019 e 2020 . No entanto, com o advento da crise pandêmica por coronavírus, houve queda no número de visitas e, consequentemente, no de clientes. Dentre as medidas encontradas nos websites, destacam-se as recomendações de saúde e a criação ou o realce de links para facilitar o acesso a anúncios que ofertam o sexo virtual. Informações sobre auxílios sociais são mais escassas.

Quanto aos anúncios analisados, menos de um por cento mencionam a crise por COVID-19. Dentre esses, há menções a descumprimento do distanciamento social; algumas medidas de proteção para o encontro offline; e oferta de sexo virtual e/ou packs com material erótico, excepcionalmente durante o período de pandemia. No entanto, a autoorganização de indivíduos e grupos é geralmente insuficiente para mitigar o impacto da pandemia por COVID-19, não devendo ser considerada como um perfeito substituto para o devido apoio governamental.

Defende-se aqui um contexto de saúde pública que reconheça a natureza emergente e mutável do trabalho sexual frente a desastres e contextos de crise, o que significa programas e políticas apropriadas para esse grupo populacional. Futuras estratégias para a promoção da saúde podem levar em consideração os códigos vigentes nos anúncios para abordagens relacionadas à realidade do mercado sexual. As iniciativas de saúde pública devem refletir e incorporar esse conhecimento. 


\section{Colaboradores}

Ambos atuaram na concepção, desenho do estudo, análise e interpretação dos dados. TS Passos redigiu o manuscrito e MA Almeida-Santos realizou a revisão crítica do artigo. Todos os autores aprovaram a versão final.

\section{Referências}

1. Adriaenssens S, Hendrickx J. Sex, price and preferences: Accounting for unsafe sexual practices in prostitution markets. Sociol Health Illn 2012; 34(5):665-680.

2. Pajnik M, Kambouri N, Renaukt M, Sorii. Digitalising sex commerce and sex work: a comparative analysis of French, Greek and Slovenian websites. Gend Place Cult 2016; 23(3):345-364.

3. Sanders T, Scoular J, Campbell R, Pitcher J, Cunningham S. Internet sex work: Beyond the gaze. Berlin: Springer; 2017.

4. DeAngelo G, Shapiro JN, Borowitz J, Cafarella M, Ré C, Shiffman G. Pricing risk in prostitution: Evidence from online sex ads. J Risk Uncertain 2019; 59(3):281305.

5. Griffiths MD, Kuss DJ, Billieux J, Pontes HM. The evolution of Internet addiction: a global perspective. Addict Behav 2016; 53:193-195.

6. Bond KT, Yoon IS, Houang ST, Downing MJ, Grov C, Hirshfield S. Transactional sex, substance use, and sexual risk: comparing pay direction for an internet-based US sample of men who have sex with men. Sex Res Social Policy 2019; 16(3):255-267.

7. Alpalhão M, Filipe P. The Impacts of Isolation Measures Against SARS-CoV-2 Infection on Sexual Health. AIDS Behav 2020; 24(8):2258-2259.

8. Guan WJ, Ni ZY, Hu Y, Liang WH, Ou CQ, He JX, Liu L, Shan H, Lei CL, Hui DS, Du B. Clinical characteristics of coronavirus disease 2019 in China. N Engl J Med 2020; 382:1708-1720.

9. Blackwell CW, Dziegielewski SF. Risk for a price: sexual activity solicitations in online male sex worker profiles. J Soc Serv Res 2013; 39(2):159-170.

10. Grov C, Rodríguez-Díaz CE, Ditmore MH, Restar A, Parsons JT. What kinds of workshops do internet-based male escorts want? Implications for prevention and health promotion. Sex Res Social Policy 2014; 11(2):176-185.

11. Sanders T, Connelly L, King LJ. On our own terms: The working conditions of internet-based sex workers in the UK. Sociol Res Online 2016; 21(4):1-14.

12. Cunningham $S$, Shah M. Decriminalizing indoor prostitution: Implications for sexual violence and public health. Rev Econ Stud 2018; 85(3):1683-1715.

13. Shehadi S, Partington M. Coronavirus: Offline sex workers forced to start again online. BBC News [Internet] 2020. [acessado 2020 Maio 26]. Disponível em: https://www.bbc.com/news/technology-52183773

14. Lumb D. Coronavirus: Sex workers 'should have access to support fund'. BBC News [Internet] 2020. [acessado 2020 Maio 26]. Disponível em: https://www.bbc.com/ news/uk-england-nottinghamshire-52579634

15. United Nations Programme on HIV/AIDS. Sex workers should not be left behind in response to COVID-19. UNAIDS [Internet] 2020. [acessado 2020 Maio 25]. Disponível em: https://www.unaids.org/en/ resources/presscentre/pressreleaseandstatementarchive/2020/april/20200408_sex-workers-covid-19

16. Passos TS, Barroso-Pavía R, Ramos NC. Género, feminización del VIH/sida y vulnerabilidad: intervención social con trabajadoras del sexo. In: Vázquez Fernández MJ, Nieto Morales C, Cordero Ramos N. Colectivos en Situación de Vulnerabilidad. Sevilla: Editorial Dykinson; 2018. p. 198-221. 
17. Barroso-Pavía R, Passos TS, Ramos NC. Trabajo sexual en Brasil y España: Análisis de las principales normas y políticas públicas. RELIES 2019; 10(1):124126.

18. Valadier C. Migração e Trabalho Sexual por uma Perspectiva de Gênero. Contexto Int 2018; 40(3):501-524.

19. Vizcaino-Suárez LP, Díaz-Carrión IA. Gender in tourism research: perspectives from Latin America. Tour Rev 2019; 74(5):1091-1103.

20. Kozinets RV. Netnography: Doing Ethnographic Research Online. Thousand Oaks: Sage publications; 2010.

21. Bardin L. Análise de conteúdo. Lisboa: Edições 70; 2010.

22. World Health Organization (WHO). Considerations for quarantine of individuals in the context of containment for coronavirus disease (COVID-19): interim guidance. Geneve: WHO; 2020.

23. Platt L, Grenfell P, Meiksin R, Elmes J, Sherman SG, Sanders T, Mwangi P, Crago AL. Associations between sex work laws and sex workers' health: A systematic review and meta-analysis of quantitative and qualitative studies. PLoS Med 2018; 15(12):1-54.

24. Jean T. The Gig is Up: Supporting Non-Standard Workers Now and After Coronavirus. Lerner Center 2020; (19):1-3.

25. Blake D. Preparedness and recovery as a privilege in the context of covid-19. Economic and Social Res Aoteaora 2020; 18:1-6.

26. Deering KN, Amin A, Shoveller J, Nesbitt A, GarciaMoreno C, Duff P, Argento E, Shannon K. A systematic review of the correlates of violence against sex workers. Am J Public Health 2014; 104:e42-54.

27. Mastrocola EL, Taylor AK, Chew-Graham C. Access to healthcare for long-term conditions in women involved in street-based prostitution: a qualitative study. BMC Fam Pract 2015; 16(1):118.

28. Howard S. Covid-19: Health needs of sex workers are being sidelined, warn agencies. BMJ 2020; 369:m1867.

29. King P, Cormack D, McLeod M, Harris R, Gurney J. 'COVID-19 and Maori health - when equity is more than a word'. Public Health Expert [Internet] 2020. [acessado 2020 Maio 25]. Disponível em: https:// blogs.otago.ac.nz/pubhealthexpert/2020/04/10/covid-19-and-maori-health-when-equity-is-more-than -a-word/
30. Fraser C, Blake D. Valuing Voices: Sex Workers' Experiences During and After the Canterbury Earthquakes. Wellington: New Zealand Prostitute's Collective \& Joint Centre for Disaster Research, Massey University; 2019.

31. Parsons JT, Koken JA, Bimbi DS. The use of the Internet by gay and bisexual male escorts: sex workers as sex educators. AIDS Care 2004; 16(8):1021-1035.

32. Pruitt MV. Online boys: male-for-male Internet escorts. Sociol Focus 2005; 38(3):189-203.

33. Brasseur P, Finez J. Performing Amateurism: a Study of Camgirls' Work. In: Naulin S., Jourdain A, editors. The Social Meaning of Extra Money. Dynamics of Virtual Work. London: Palgrave Macmillan; 2020. p. 211237.

34. Dong E, Du H, Gardner L. An interactive web-based dashboard to track COVID-19 in real time. Lancet Infect Dis 2020; 20(5):533-534.

35. Kille J, Bungay V, Oliffe J, Atchison C. A content analysis of health and safety communications among Internet-based sex work advertisements: important information for public health. J Med Internet Res 2017; 19(4):e111.

Artigo apresentado em 05/06/2020

Aprovado em 25/08/2020

Versão final apresentada em 27/08/2020 